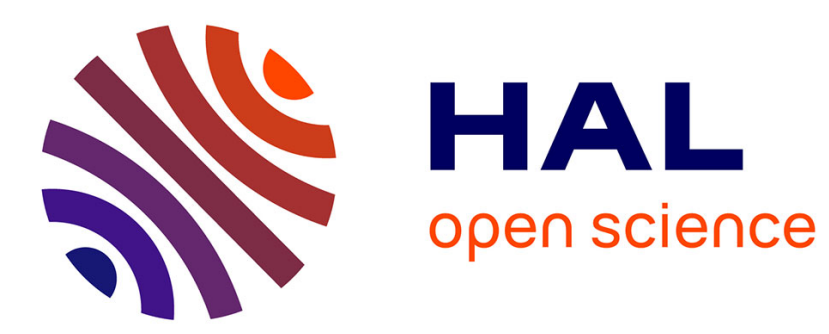

\title{
Energy-loss magnetic chiral dichroism study of epitaxial MnAs film on $\operatorname{GaAs}(001)$
}

Xiaoxiao Fu, Bénédicte Warot-Fonrose, Rémi Arras, D. Demaille, M. Eddrief, Victor H. Etgens, Virginie Serin

\section{- To cite this version:}

Xiaoxiao Fu, Bénédicte Warot-Fonrose, Rémi Arras, D. Demaille, M. Eddrief, et al.. Energy-loss magnetic chiral dichroism study of epitaxial MnAs film on GaAs(001). Applied Physics Letters, 2015, 107 (6), pp.062402. 10.1063/1.4928542 . hal-01442943

\section{HAL Id: hal-01442943 \\ https://hal.science/hal-01442943}

Submitted on 7 Mar 2018

HAL is a multi-disciplinary open access archive for the deposit and dissemination of scientific research documents, whether they are published or not. The documents may come from teaching and research institutions in France or abroad, or from public or private research centers.
L'archive ouverte pluridisciplinaire HAL, est destinée au dépôt et à la diffusion de documents scientifiques de niveau recherche, publiés ou non, émanant des établissements d'enseignement et de recherche français ou étrangers, des laboratoires publics ou privés. 


\section{Energy-loss magnetic chiral dichroism study of epitaxial MnAs film on GaAs(001)}

X. Fu, B. Warot-Fonrose, R. Arras, D. Demaille, M. Eddrief, V. Etgens, and V. Serin

Citation: Appl. Phys. Lett. 107, 062402 (2015); doi: 10.1063/1.4928542

View online: https://doi.org/10.1063/1.4928542

View Table of Contents: http://aip.scitation.org/toc/apl/107/6

Published by the American Institute of Physics

\section{Articles you may be interested in}

Determination of magnetic parameters in $\mathrm{La}_{0.7} \mathrm{Sr}_{0.3} \mathrm{MnO}_{3} / \mathrm{SrTiO}_{3}$ thin films using EMCD

Applied Physics Letters 108, 242414 (2016); 10.1063/1.4954168

Circular dichroism in the electron microscope: Progress and applications (invited)

Journal of Applied Physics 107, 09D311 (2010); 10.1063/1.3365517

Energy loss magnetic chiral dichroism: A new technique for the study of magnetic properties in the electron microscope (invited)

Journal of Applied Physics 103, 07D931 (2008); 10.1063/1.2836680

From molecules to solids with the $\mathrm{DMol}^{3}$ approach

The Journal of Chemical Physics 113, 7756 (2000); 10.1063/1.1316015

First-principles study of the stability of free-standing germanene in oxygen atmosphere

Journal of Applied Physics 118, 124303 (2015); 10.1063/1.4931057

Combined effects of vertical and lateral confinement on the magnetic properties of MnAs micro and nano-ribbons Journal of Applied Physics 120, 093905 (2016); 10.1063/1.4961501

\section{Scilight}

Sharp, quick summaries illuminating the latest physics research

\section{Sign up for FREE!}

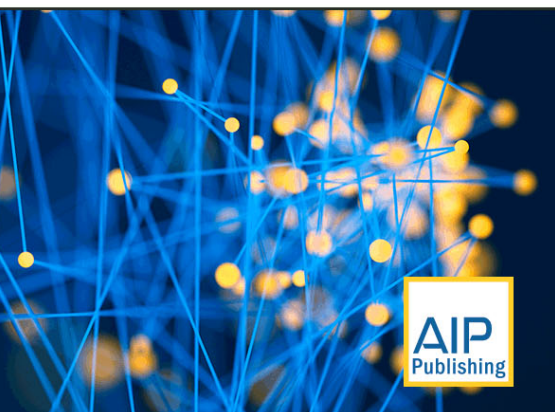




\title{
Energy-loss magnetic chiral dichroism study of epitaxial MnAs film on $\operatorname{GaAs}(001)$
}

\author{
X. Fu, ${ }^{1}$ B. Warot-Fonrose,${ }^{1}$ R. Arras,${ }^{1}$ D. Demaille, ${ }^{2,3}$ M. Eddrief, ${ }^{2,3}$ V. Etgens,${ }^{2,3,4}$ \\ and V. Serin ${ }^{1}$ \\ ${ }^{1}$ CEMES-CNRS UPR 8011, Univ. Toulouse, F-31055 Toulouse, France \\ ${ }^{2}$ Sorbonne Universités, UPMC Univ. Paris 6, UMR 7588, INSP, 4 Place Jussieu, F-75005 Paris, France \\ ${ }^{3}$ CNRS, UMR 7588, Institut des Nanosciences de Paris, 4 Place Jussieu, F-75005 Paris, France \\ ${ }^{4}$ Institut VeDeCoM - UVSQ, 78000 Versailles, France
}

(Received 10 June 2015; accepted 1 August 2015; published online 12 August 2015)

\begin{abstract}
The room-temperature ferromagnetic behavior of $\mathrm{MnAs} / \mathrm{GaAs}(001)$ thin film has been locally explored by Transmission Electron Microscope (TEM). We first differentiated hexagonal $\alpha$-MnAs and quasi-hexagonal $\beta$-MnAs which are very similar in atomic structure by electron diffraction. Local magnetic moment information of the identified $\alpha$-MnAs was extracted from manganese- $\mathrm{L}_{2,3}$ edges using Energy-loss Magnetic Circular Dichroism technique and the ratio of orbital to spin magnetic moment was measured. In this experiment, atomic structure identification, chemical analysis, and magnetic moment measurement were simultaneously achieved at high spatial resolution in TEM, thus providing a potential method for in-situ study of local properties of multiphase magnetic materials. @ 2015 AIP Publishing LLC. [http://dx.doi.org/10.1063/1.4928542]
\end{abstract}

Epitaxial MnAs thin films grown on GaAs substrate are of high interest due to its potential applications in spin injection, ${ }^{1-3}$ magnetic tunnel junction, ${ }^{4,5}$ and magnetologic devices. ${ }^{6}$ The magnetization, as one of the most essential magnetic properties, has been intensively investigated. ${ }^{7}$ Techniques such as cantilever beam magnetometer $(\mathrm{CBM}){ }^{8}$ superconducting quantum interference device (SQUID), ${ }^{9-11}$ and X-ray magnetic circular dichroism $(\mathrm{XMCD})^{12}$ have been applied to quantify its magnetization over the temperature range from $0 \mathrm{~K}$ to $400 \mathrm{~K}$. Generally, magnetization investigated at room-temperature (RT), the common working temperature of devices in practical applications, is of special concern. However at RT, there is a phase coexistence of ferromagnetic $\alpha$-MnAs and paramagnetic $\beta$-MnAs for at least $30 \mathrm{~K}$ below Curie temperature (approximately $313 \mathrm{~K}) .^{7}$ The published magnetization or magnetic moment may include the contribution of $\alpha$-MnAs crystals with various orientations and with crystallographic defects, or the contribution of both $\alpha$ - and $\beta$-MnAs in a coexistence region, due to the limited spatial resolution of these techniques.

The emerging technique Electron energy-loss Magnetic Chiral Dichroism (EMCD), based on transmission electron microscope (TEM) with spatial resolution down to $2 \mathrm{~nm},{ }^{13,14}$ provides a powerful method to extract local magnetic moment information of ferromagnetic $\alpha$-MnAs. As in the case of XMCD, EMCD is not only able to extract the magnetic moment information of specific element but also the respective contribution of orbital and spin moment. ${ }^{15,16}$ Moreover, it allows to access simultaneously to the atomic structure, to the chemical component, and to the magnetic moment in TEM, facilitating in-situ study of magnetic crystals. In the relative work reported until now, $\mathrm{Fe}$ and $\mathrm{Co}_{2,3}$ edges have been extensively studied, ${ }^{13,16-23}$ but MnAs thin films have not been tested so far with this technique.

In this letter, $\mathrm{MnAs} / \mathrm{GaAs}(001)$ was investigated at RT by TEM techniques. Electron diffraction (ED) was applied for the identification of the $\alpha$-MnAs crystal in the coexistence region, and EMCD was used for local quantitative magnetic moment information. The measured $m_{l} / m_{s}$ ratio is compared with values obtained, thanks to first principles calculations based on the density functional theory (DFT).

A 100-nm MnAs layer was epitaxially grown on a $\operatorname{GaAs}(001)$ substrate, using solid-source molecular beamepitaxy (MBE). Growth details are given elsewhere. ${ }^{24}$ The microscopy experiments were performed on the SACTEMToulouse, a Tecnai F20 microscope (operating at $200 \mathrm{kV}$ ), fitted with an objective lens aberration corrector (Corrected electron optical systems (CEOS)) and imaging filter (Gatan Tridiem). Plan-view sample was prepared by polishing from the substrate side until the thickness of $10 \mu \mathrm{m}$ and then made transparent by ion-beam milling (Precision Ion Polishing System (PIPS)-Gatan). The theoretical DFT calculations were performed with the full-potential linearized augmented plane waves code Wien $2 \mathrm{k}^{25}$ We used the generalized gradient approximation $(\mathrm{GGA})^{26}$ for the exchange-correlation functional. The atomic sphere radii were chosen to 2.0 atomic units for both $\mathrm{Mn}$ and As, and we made the calculations with the following experimental parameters $\mathrm{a}=3.7253$ $\dot{\mathrm{A}}$ and $\mathrm{c}=5.7031 \dot{\mathrm{A}}$. The irreducible part of the first Brillouin zone was sampled with $1464 \mathrm{k}$-points and the convergence parameter RKmax was set to 7.0. The $m_{l}$ and $m_{s}$ moments were calculated for the d-electrons of the Mn atom, with and without an additional orbital potential. Spin-orbit interaction has been added in the [11 $\overline{2} 0]$ direction.

It is known that $\alpha$-MnAs crystallizes in the hexagonal NiAs-type $\left(\mathrm{B}_{1}\right)$ structure, and $\beta$-MnAs in the orthorhombic MnP-type (B31) structure. ${ }^{27}$ In fact, the lattice of $\beta$-MnAs corresponds to the slightly distorted $\alpha$-MnAs lattice, thus the $\beta$-MnAs is also considered as quasi-hexagonal. ${ }^{27,28}$ Figs. 1(a) and 1(b) demonstrate the similarity as well as the tiny difference between atomic structures of the two phases. It was once reported that the boundary between the two phases could not be resolved in HRTEM images. ${ }^{7}$ Unfortunately, as 

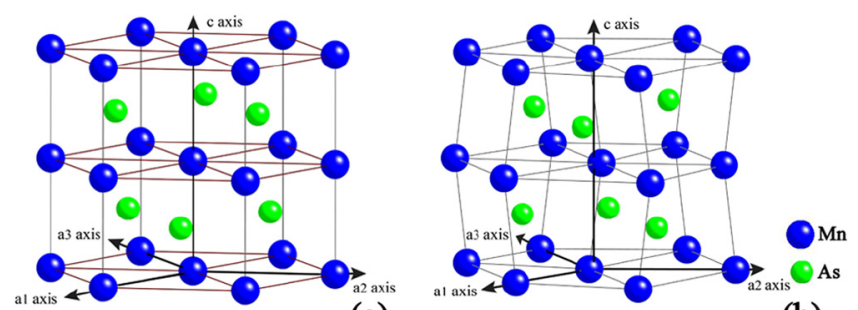

(a)
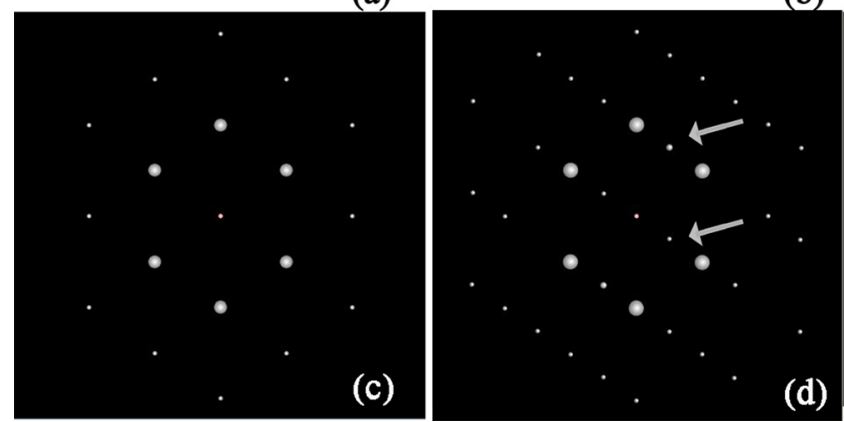

FIG. 1. Atomic structure of hexagonal $\alpha$-MnAs (a) and orthorhombic $\beta$-MnAs (b). Diffraction patterns of $\alpha$-MnAs at $\langle\overline{2} 201\rangle$ zone axes in (c) and that of $\beta$-MnAs at the zone axis in (d). The diffraction patterns are simulated by PDF software.

a result of simulation, their diffraction patterns are also too similar to differentiate at most zone axes in practical cases. However, because of the regular distortions in specific directions, though small, electron diffraction patterns of $\beta$-MnAs at some specific zone axes may differ from the patterns of $\alpha$-MnAs. One example is demonstrated in Figs. 1(c) and 1(d). We apply four-parameter Miller-Bravais indices to the quasi-hexagonal $\beta$-MnAs crystal, with tolerance of small atom displacement due to lattice distortion, to follow the indices of hexagonal $\alpha$-MnAs. In the hexagonal $\alpha$-MnAs crystal, there are six symmetrical lattice orientations at an angle of $66.10^{\circ}$ relative to [0001], which are

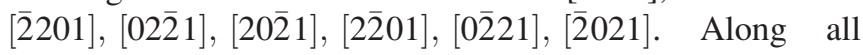
these six symmetrical zone axes, the diffraction patterns of $\alpha$-MnAs are nearly the same as seen in Fig. 1(c). However, except for the similar spots in Fig. 1(c), some extra spots must appear in the diffraction pattern of $\beta$-MnAs at the same zone axes. One case is as seen in Fig. 1(d). If the zone axis in Fig. 1(c) is set to be [ 2201$]$, the extra spots in Fig. 1(d) originate from a longer-range order that appears due to the displacement of atoms in (10 $\overline{1} 2)$ lattice plane to opposite directions alternatively. In the other case, which will not be shown here, the extra spots appear in different positions from Fig. 1(d) due to the asymmetry of atomic structure of $\beta$-MnAs. That is to say, that the pattern in Fig. 1(c) without extra spots is unique for $\alpha$-MnAs. In addition, the zone axis of [2201], as well as its crystallographic direction families, is not the only possibility to achieve the structure differentiation.

Several in-plane and out-of-plane epitaxial orientation of MnAs films on $\mathrm{GaAs}(001)$ substrate have been previously observed, ${ }^{7,12,24}$ with the $c$-axis in-plane and tilted out-ofplane with respect to the GaAs(100) surface, so-called ( $\left.A_{0}, B_{0}\right)$-type and $\left(A_{1}, A_{2}, B_{1}, B_{2}, \ldots\right)$-type, respectively. ${ }^{7,12}$ The surface template in the initial growth plays a critical role in crystal orientation of MnAs epitaxial layer on GaAs (001). For this EMCD study, the MnAs film has c-axis tilted with respect to the $\mathrm{GaAs}(001)$ surface (type- $A_{1}, A_{2}, B_{1}, B_{2}, \ldots, 12$ ), according to the cross-sectional TEM image (not reported here). The local magnetic information of MnAs epilayer was investigated in the plan-view sample, on a grain of single MnAs crystal-orientation with a diameter of around $200 \mathrm{~nm}$ as shown in Fig. 2(a). The grain was orientated to the specific zone axis [22̄01], and a diffraction pattern in the inset of Fig. 2(a) on the selected grain was acquired using selected area electron diffraction (SAED) technique. The diffraction pattern, as explained above, demonstrates that only the hexagonal $\alpha$-MnAs phase is present in this grain.

Electron energy-loss spectroscopy (EELS) was then used to check the thickness and the elemental composition of the selected crystal. The relative sample thickness $t / \lambda, \lambda$ being the inelastic mean free path, is measured to be 0.41 . No carbon and a very small amount of oxygen, which reveals negligible traces of contaminations, have been detected by EELS. Fig. 2(b) shows an EELS spectrum, with energy loss ranging from $500 \mathrm{eV}$ to $1450 \mathrm{eV}$. Mn- $\mathrm{L}_{2,3}$ and $\mathrm{As}-\mathrm{L}_{2,3}$ edges, respectively, at $640 \mathrm{eV}$ and $1323 \mathrm{eV}$ are shown in the spectrum. No Ga edge has been detected. It means that the GaAs substrate layer in the plan-view sample is totally polished away, and only MnAs layer is preserved in this region. The
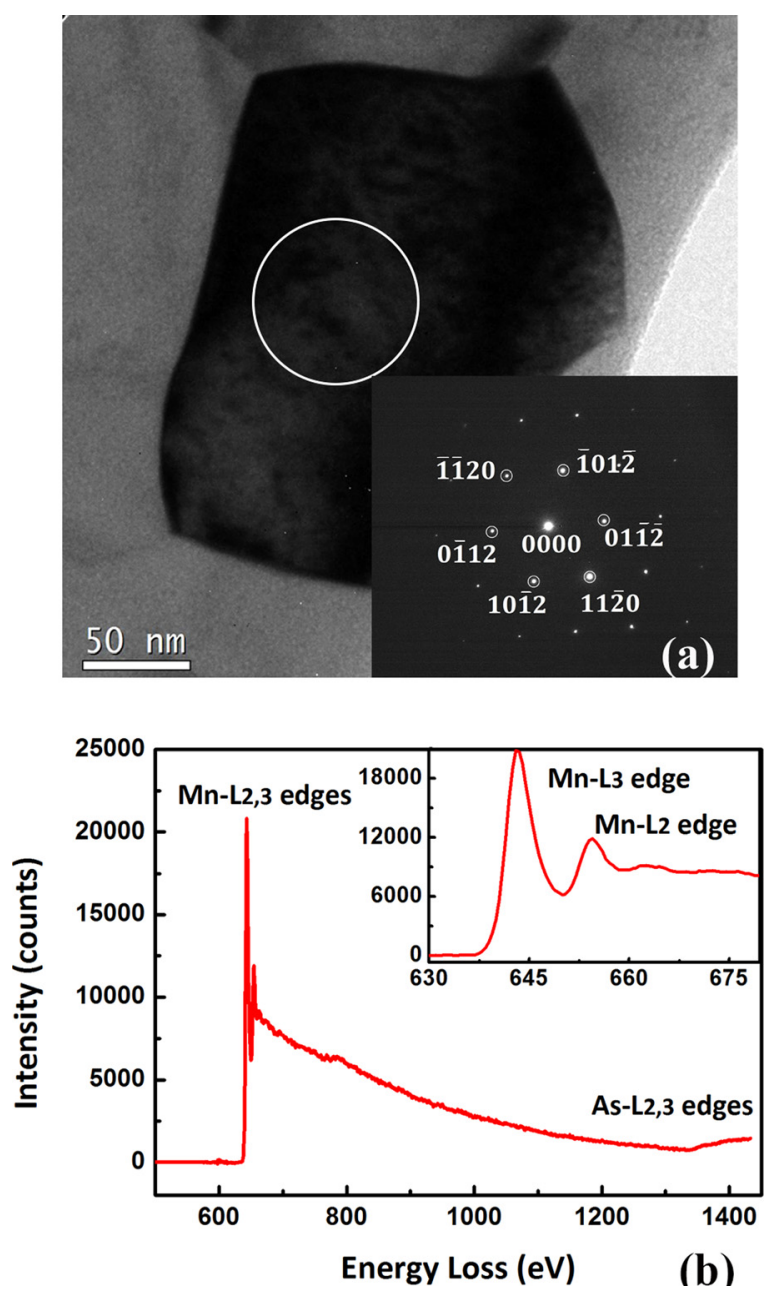

FIG. 2. (a) Bright field image of selected grain in the plan-view sample of $\mathrm{MnAs} / \mathrm{GaAs}(001)$ at RT at low magnification of $60000 \times$, and diffraction pattern acquired at zone axis of [ $\overline{2} 201]$ in the inset. (b) Electron energy loss near edge spectrum of the crystal at RT. 
amplified near edge fine structure of $\mathrm{Mn}-\mathrm{L}_{2,3}$, edges is shown in the inset of Fig. 2(b).

Then, EMCD technique has been applied on the same grain for which the phase, thickness, and elemental composition have been previously specified. We used Large-Angle Convergent Diffraction (LACDIF) experimental setup to obtain a diffraction pattern in image plane ${ }^{23}$ and applied Energy spectrum imaging (ESI) technique ${ }^{23,29}$ to record a data cube. The spatial resolution reaches a few tens of nanometers. The MnAs layer was saturated along the beam axis by the magnetic field as large as $2 \mathrm{~T}$ coming from objective lens. The crystal was orientated in two-beam configuration to minimize the n-beam dynamic interaction, in which only diffraction spot $(11 \overline{2} 0)$ was excited. From the corrected data cube, EELS spectra were extracted in positions 1 and 2, pointed in the inset of Fig. 3. In the two symmetrical points on the Thales circles of the diffraction plane, different combinations of two electron scattering vectors give rise to left- and right-handed polarized virtual photons, which are analog to circular polarized X-ray in XMCD. The EELS spectra detected in the two positions carry the magnetic moment information. Two typical EELS spectra shown in Fig. 3 were extracted using circular apertures with normalized diameter of 0.5 . The values of the signal difference around L2 and L3 edge peak are opposite in sign, confirming magnetism of the detected sample.

We now apply sum rule ${ }^{15,16}$ to EMCD spectra for quantitative analysis. Formula in sum rules is available to calculate orbital and spin moments of magnetic samples from the integrated areas under $\mathrm{L}_{2}$ and $\mathrm{L}_{3}$ edge curves in EMCD spectra. The $m_{l} / m_{s}$ ratio can be extracted straightforwardly when the scattering conditions are properly set. In our case, the spectra were integrated within the energy windows [640 $\mathrm{eV}-650 \mathrm{eV}$ ] for $\mathrm{Mn}-\mathrm{L}_{3}$ edge and [652 eV-660 eV] for Mn$\mathrm{L}_{2}$ edge. The two windows were selected to cover the whole ranges of L2 and L3 edges, respectively. It is known that the signal obtained with EMCD technique depends sensitively on many factors, such as sample thickness, detector position, and aperture size. But for the ratio of orbital moment to spin moment, the measurement result is not affected by these factors. ${ }^{15}$ The ratio of orbital moment to spin moment was measured to be $0.07 \pm 0.05$. There are three main sources of statistical error. The first one is the relatively low signal-to-

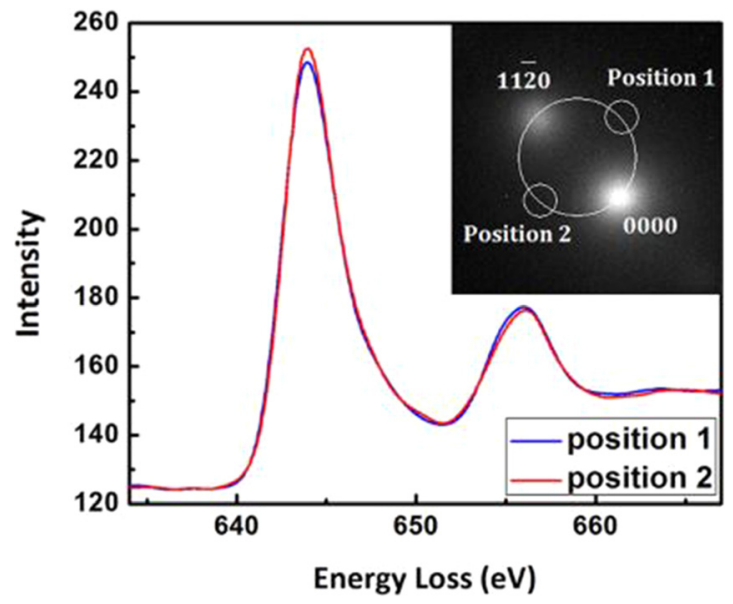

FIG. 3. EELS spectra acquired on ferromagnetic MnAs at Mn-L2,3 edge, extracted from position 1 and position 2 shown in the inset. noise ratio. The second one is the sample movement, which is a tricky problem in TEM and cannot be corrected in post data treatment. Moreover, the exactly same two-beam condition is hard to obtain all the time, and small tilt from that condition can influence the result. The magnetic moment ratio of Mn atom in MnAs measured by EMCD technique has not been reported before. Recently, a lower value of around 0.03 has been published for $\mathrm{MnAs} / \mathrm{GaAs}(001)$ at RT with XMCD. ${ }^{12}$ The comparison is, however, not straightforward between the two values. Primarily, the epitaxial planes of the two measured MnAs layers on $\mathrm{GaAs}(001)$ are not the same. Second, the XMCD result is a value after correction of spin moment with a constant of 1.47 , in order to compensate the effect of the mixing between $\mathrm{L}_{2}$ and $\mathrm{L}_{3}$ edges. ${ }^{12}$ Our DFT calculations give a value even lower, of 0.008 or 0.011 , respectively, without or with the additional orbital potential. The theoretical calculation is based on the atomic ground state at $\mathrm{T}=0 \mathrm{~K}$. But at RT, other $|\mathrm{JM}|$ states can mix into the ground state. In addition, we have to note that the determination of magnetic moment is still on the way to be more accurate. The $m_{l} / m_{s}$ ratio of $\mathrm{Fe}$, as an example, obtained with EMCD in literature varies from 0.024 to $0.09^{13,17,18,20,21}$ and XMCD results vary from 0.043 to $0.12 .^{18}$ They are somewhat larger than the theoretical value of $0.021 .^{30}$ But it is of interest and value to make quantitative measurement with EMCD on varied ferromagnetic materials to discuss its potential applicability and improvement.

In summary, we performed experiments within TEM to acquire the atomic structure, chemical components, and local magnetic moment information on exactly the same ferromagnetic $\alpha$-MnAs crystal. In the MnAs thin film with an outof-plane grown axis on $\operatorname{GaAs}(001)$ substrate, we identified the hexagonal $\alpha$-MnAs crystal in the coexistence region of $\alpha$-MnAs and $\beta$-MnAs at RT by electron diffraction technique. Then, EELS was applied to check the chemical components of the selected crystal, in order to exclude the interference of contamination or oxidation layer to the following experiment. Finally, the ratio of orbital and spin magnetic moment of Mn atom was obtained from the $\alpha$-MnAs crystal with EMCD technique at high spatial resolution of a few tens of nanometers. It provides a method for future in-situ study of local properties of epitaxial MnAs thin film, as well as a method to study the local magnetic properties corresponding to each phase in some multiphase coexistence situation. Higher signal to noise ratio and more efficient sum rule are necessary for more accurate determination of magnetic moment with EMCD technique in the future application.

This work was supported by the French national project EMMA (ANR12 BS10 013 01) and by the European Union under the Seventh Framework Program under a contract for an Integrated Infrastructure Initiative Reference 312483ESTEEM2. The authors would like to thank S. Cherifi for fruitful discussions and C. Crestou for her valuable help in the sample preparation.

${ }^{1}$ E. D. Fraser, S. Hegde, L. Schweidenback, A. H. Russ, A. Petrou, H. Luo, and G. Kioseoglou, Appl. Phys. Lett. 97, 041103 (2010).

${ }^{2}$ L. Däweritz, M. Kästner, T. Hesjedal, T. Plake, B. Jenichen, and K. H. Ploog, J. Cryst. Growth 251, 297-302 (2003). 
${ }^{3}$ K. H. Ploog, J. Appl. Phys. 91, 7256 (2002).

${ }^{4}$ S. Sugahara and M. Tanaka, Appl. Phys. Lett. 80, 1969-1971 (2002).

${ }^{5}$ V. Garcia, H. Jaffrès, M. Eddrief, M. Marangolo, V. H. Etgens, and J. M. George, Phys. Rev. B 72, 081303 (2005).

${ }^{6}$ C. Pampuch, A. K. Das, A. Ney, L. Däweritz, R. Koch, and K. H. Ploog, Phys. Rev. Lett. 91, 147203 (2003).

${ }^{7}$ L. Däweritz, Rep. Prog. Phys. 69, 2581 (2006).

${ }^{8}$ A. K. Das, C. Pampuch, A. Ney, T. Hesjedal, L. Däweritz, R. Koch, and K. H. Ploog, Phys. Rev. Lett. 91, 087203 (2003).

${ }^{9}$ J. Kim, H. Akinaga, and J. Kim, Appl. Phys. Lett. 98, 102511 (2011).

${ }^{10}$ L. Däweritz, C. Herrmann, J. Mohanty, T. Hesjedal, K. H. Ploog, E. Bauer, A. Locatelli, S. Cherifi, R. Belkhou, A. Pavlovska, and S. Heun, J. Vac. Sci. Technol. 23, 1759-1768 (2005).

${ }^{11}$ V. Z. C. Paes, I. L. Graff, J. Varalda, V. H. Etgens, and D. H. Mosca, J. Phys.: Condens. Matter 25, 046003 (2013).

${ }^{12}$ J. M. Wikberg, R. Knut, S. Bhandary, I. di Marco, M. Ottosson, J. Sadowski, B. Sanyal, P. Palmgren, C. W. Tai, O. Eriksson, O. Karis, and P. Svedlindh, Phys. Rev. B 83, 024417 (2011).

${ }^{13}$ P. Schattschneider, S. Rubino, C. Hébert, J. Rusz, J. Kuneš, P. Novák, E. Carlino, M. Fabrizioli, G. Panaccione, and G. Rossi, Nature 441, 486-488 (2006).

${ }^{14}$ P. Schattschneider, M. Stöger-Pollach, S. Rubino, M. Sperl, C. Hurm, J. Zweck, and J. Rusz, Phys. Rev. B 78, 104413 (2008).

${ }^{15}$ L. Calmels, F. Houdellier, B. Warot-Fonrose, C. Gatel, M. J. Hÿtch, V. Serin, E. Snoeck, and P. Schattschneider, Phys. Rev. B 76, 060409 (2007).

${ }^{16}$ J. Rusz, O. Eriksson, P. Novák, and P. M. Oppeneer, Phys. Rev. B 76, 060408 (2007).
${ }^{17}$ Z. Q. Wang, X. Y. Zhong, R. Yu, Z. Y. Cheng, and J. Zhu, Nat. Commun. 4, 1395 (2013).

${ }^{18}$ H. Lidbaum, J. Rusz, A. Liebig, B. Hjörvarsson, P. M. Oppeneer, E. Coronel, O. Eriksson, and K. Leifer, Phys. Rev. Lett. 102, 037201 (2009).

${ }^{19}$ B. Warot-Fonrose, C. Gatel, L. Calmels, V. Serin, E. Snoeck, and S. Cherifi, J. Appl. Phys. 107, 09D301 (2010).

${ }^{20}$ B. Warot-Fonrose, C. Gatel, L. Calmels, V. Serin, and P. Schattschneider, Ultramicroscopy 110, 1033-1037 (2010).

${ }^{21}$ J. Rusz, H. Lidbaum, S. Rubino, B. Hjörvarsson, P. M. Oppeneer, O. Eriksson, and K. Leifer, Phys. Rev. B 83, 132402 (2011).

${ }^{22}$ S. Muto, J. Rusz, K. Tatsumi, R. Adam, S. Arai, V. Kocevski, P. M. Oppeneer, D. E. Bürgler, and C. M. Schneider, Nat. Commun. 5, 3138 (2014).

${ }^{23}$ B. Warot-Fonrose, F. Houdellier, M. J. Hÿtch, L. Calmels, V. Serin, and E. Snoeck, Ultramicroscopy 108, 393-398 (2008).

${ }^{24}$ R. Breitwieser, F. Vida, I. L. Graff, M. Marangolo, M. Eddrief, J. C. Boulliard, and V. H. Etgens, Phys. Rev. B 80, 045403 (2009).

${ }^{25}$ P. Blaha, K. Schwarz, G. K. H. Madsen, D. Kvasnicka, and J. Luitz, WIEN2K, Vienna University of Technology, Vienna, 2001.

${ }^{26}$ J. P. Perdew, K. Burke, M. Ernzerhof, J. P. Perdew, K. Burke, and M. Ernzerhof, "Generalized gradient approximation made simple," Phys. Rev. Lett. 77, 3865-3868 (1996).

${ }^{27}$ R. H. Wilson and J. S. Kasper, Acta Crystallogr. 17, 95-101 (1964).

${ }^{28}$ I. Rungger and S. Sanvito, Phys. Rev. B 74, 024429 (2006).

${ }^{29}$ C. Gatel, B. Warot-Fonrose, and P. Schattschneider, Ultramicroscopy 109, 1465-1471 (2009).

${ }^{30}$ S. Muto, K. Tatsumi, and J. Rusz, Ultramicroscopy 125, 89-96 (2013). 